\title{
RESEÑAS DE CONGRESOS Y JORNADAS
}

XXXVII REUNION INTERNACIONAL DE LA C.I.E.A.E.M.: MATEMATICAS PARA TODOS EN LA ERA DE LOS ORDENADORES

En el volumen 1, n०3 (noviembre del 83) de esta misma revista, haciamos la reseña de la anterior reunión pública de la CIEAEM, celebrada en Lisboa.

La reunion $n^{\circ}$ XXXVI tuvo un caracter interno, exclusivo para los miembros de la comisión, se celebró en Frascati (Italia), entre los días 16 y 20 de abril del año 84, dedicándose la misma, al estudio y mejora no de la ensettanza matemática, sino de la organización de las reuniones públicas, para que las mismas sean más fructíferas. En esta reunión, y provisionalmente, se nombró presidente a Hans Freudenthal, que propuso celebrar la siguiente reunión pública en su país.

Por ello, la XXXVII reunión se ha celebrado en Leyden, organizada y patrocinada principalmente por el OW \& OC (Wiskundeonderwijs \& onderwijs Computercentrum), sucesor del ya desaparecido IOWO.

El tema, en torno al cual se debian presentar las comunicaciones era «Matemáticas para todos en la era de los ordenadores", proponiéndose cinco subtemas para mejor llevar a cabo el trabajo de la reunión: El grupo I era de Cálculo: aritmético y algebraico. El grupo 2: Modelos matemáticos. Grupo 3: Razonamiento matemático. Grupo 4: Formación de profesores. Grupo 5: Matemáticas e Informática.

El trabajo de la reunión se dividió en tres grandes grupos:

- Conferencias de carácter general.

- Presentación de comunicaciones a cada uno de los subtemas de la reunión; siete se presentaron al grupo 1 , doce a la del grupo 2, catorce para el grupo 3, trece al grupo 4, doce al grupo 5 y siete de carácter diverso.

- Discusión en grupos de trabajo del conjunto de comunicaciones presentadas a cada uno de los cinco temas, para, con la aportación de todos los asistentes, obtener algunas conclusiones provisionales, que luego se pasaron a una sesión plenaria de todos los asistentes, para, tras unas nuevas discusiones por grupos de trabajo, someter a la asamblea del último dia las conclusiones definitivas de cada uno de ellos.

Las actas se pueden solicjtar a:

Groupe de Recherche OW \& OC

Tiberdreef 4

NL - 3561 GG UTRECHT (Pays-Bas)

F. Villarroya

JORNADAS DE ESTUDIO SOBRE LA INVESTIGACION EN LA ES. CUELA (Sevilla 1985)

\section{Introducción}

Toda práctica educativa obedece, de manera más o menos explícita, a un cierto modelo đidáctico. Haciendo un somero análisis de la realidad educativa en nuestro pais, se puede afirmar con bastante seguridad que un número mayoritario de profesionales de la enseñanza organizan su actividad docente guiados por una secuencia de contenidos temáticos (en gran parte inspirados en los libros de texto) o, en el mejor de los casos, por complicados sistemas de objetivos que acaban convirtièndose en un penoso proceso aburocratizado» de planificar la enseñanza.

Por otro lado, existe también un buen número de profesionales que al rechazar las estrategias anteriores e intentar liberarse de las rigideces de sus planteamientos, se balancean hacia posiciones «espontaneistas» y «practicistas» que, si bien producen cambios de interés en cuanto al uso de técnicas y recursos didácticos, suelen adolecer de una adecuada relación metodológica entre la teoría pedagógica y la práctica concreta que provoca frecuentemente la desorientación y el desánimo.

Los dos primeros casos expuestos, e! basado en los contenidos y el basado en los objetivos, obedecen, sin duda alguna, a modelos dídácticos definidos que implican determinadas concepciones teóricas e ideológicas con res- pecto a los procesos de ensef̃anzaaprendizaje. Esto no implica que los profesionales acogidos a dichas prác ticas sean siempre conscientes de ello.

Por el contrario, los diversos tanteos re novadores que desde hace tiempo se vienen produciendo en nuestro país, adolecen de modelos teóricos con el potencial suficiente como para dinamizar y orientar, de manera explícita, la acción educativa de sectores importantes de la enseñanza. Existen, eso sí, diversas aportaciones que sustentadas en as pectos parciales de la psicología, sociología 0 , en otros casos, de la filosofia educativa, son ya instrumentos importantes que nos acercan a dichos «modelos" de referencia.

En otro orden de cosas, nos parece importante resaltar el proceso de reformas institucionales en que está inmerso, a distintos niveles, el actual sistema educativo. Este proceso despierta inevitablemente numerosas expectativas, de uno u otro signo, sobre el sentido y la naturaleza de las mismas.

Estas son algunas de las cuestiones que llevaron a plantearnos, ante esta $3^{a}$. edición, si es posible trabajar en nuestro pais hacia un nuevo modelo didactico, construido desde la sintesis de diversas aportaciones teorico-prácticas, basado quizás en la Investigación en la Escuela:

- como proceso de construcción del saber por parte de los estudiantes

- como forma de acceder a un conocimiento más científico del aula por parte de los enseñantes, y

- como método para desarroilar un curriculum didáctico en construcción y reformulación permanente.

\section{Participantes}

La coordinación general ha corrido a cargo de Pedro Cañal de León y Rafael Porlán Ariza, profesores de la E.U. de Magisterio de Sevilla, contando con el patrocinio y el apoyo activo de la Consejería de Educación de la Junta de Andalucía, por medio de la Dirección General de Promoción Educativa y Renovación Pedagógica. Debe mencio- 
narse asimismo, el aporte técnico y de infraestructura de la E.U. de Magisterio de Sevilla, de la Asociación Acción Educativa de Sevilla (Convivir con la Naturaleza) y del Colectivo Andaluz de Pedagogía Popular.

En to que se refjere a los matriculados, debemos decir que las previsiones de participación se han visto ampliamente sobrepasadas. El total ce asistentes ha superado las 600 personas, de las cuales 563 formalizaron su matrícula. El $82,6 \%$ procedía de Andalucía y el resto de las diversas Comunidades Autónomas.

\section{Contenido de las jomadas}

Las Jomadas se han desarrollado en torno a cuatro tipos de actividades: ponencias generales, ponencias simultáneas, comunicaciones y seminarios de trabajo.

Las ponencias generales reflejadas en el libro de ponencias y comunicaciones, fueron las siguientes:

- 12 Ponencia.- Los conceptos estructurantes en el aprendizaje por investigación, por Raúl Gagliardi.

- $2^{2}$ Ponencia.- Aproximación a un enfoque integrador para el análisis de la actividad escolar, por Amelia Alvarez y Pablo del Rfo.

- $3^{\mathrm{a}}$ Ponencia.- El maestro investigador y la reforma curricular, por John Elliott.

Por otro lado, las ponencias simultáneas fueron:

- Escuela, Territorio y Cultura: ámbitos de la educación, por Fabricio Caivano.

- La conducta exploratoria y la investigación del niño, por Rafael Moreno.

- Los errores conceptuales como ori- gen de un nuevo modelo didáctico: de la búsqueda a la investigación, por $\mathrm{Da}$ niel Gil.

- La formación inicial del profesorado en la investigación, por Francisco Imbernón.

- El maestro como investigador en el aula. Investigar para conocer, conocer para enseñar, por Rafael Porlán.

- Aportaciones desde la Psicología cognitiva a la Investigación en la escuela, por Asunción López.

- El mėtodo cientifico y la investigación del nirto, por J. Eduardo García.

- Un enfoque curricular basado en la Investigación, por Pedro Cantal.

En cuanto a las Comunicaciones, la Comisión Asesora selecciono 55 de las 87 recibidas, que fueron distribuidas en los siguientes grupos temáticos: aspectos teóricos sobre la investigación en la escuela; Área de Ciencias de la Naturaleza; Formación inicial del profesor en la metodología investigativa; Estudios sobre el profesor; Area de Ciencias Sociales; Recursos didácticos y Áreas de Expresión.

Paralelamente a la exposición de las co municaciones, se constituyó un seminario de trabajo en el que participaron Raúl Gagliardi, Amelia Alvarez y Pablo del Río, Fabricio Caivano, Rafael Moreno, Daniel Gil, Francisco Imbernón, Asunción López, J. Eduardo García, Rafael Porlán y Pedro Cañal. En dicho seminario se analizaron las aportaciones de cada uno de los participantes con el fin de ir esbozando un modelo didáctico alternativo basado en la investigación en la escuela. Como fruto de las sesiones se estableció un calendario de trabajo para los meses venideros y un compromiso de publicar en común los resultados del mismo.
Para concluir este apartado, hemos de referimos a la intervención del profesor Antonio Rodríguez Almodóvar, Director General de Promoción Educativa y Renovación Pedagógica, en el acto de inauguración de estas III Jornadas, en la que hizo referencia a la situacion actual de la Reforma y renovación de las Ensentanzas Bástcas en Andalucía y su relación con la investigación en la escuela.

\section{Valoración final}

En primer lugar, queremos destacar el interés que ha despertado esta tercera convocatoria, habiendo aumentado considerablemente el número de participantes.

Consideramos que ha sido importante el número y calidad de los ponentes que constituyen un núcleo representativo de lo que se está haciendo en Espaffa en relación con el tema.

Valoramos positivamente también el elevado número de comuricaciones que se nos ha remitido, habiendo mejorado el nivel de las mismas y su grado de adecuación al contenido específico de las Jornadas.

Por otro lado, se ha introducido una nueva vertiente en las Jornadas a través del seminario constituido para trabajar en profundidad el lema de este afo: hacia un nuevo modelo didáctico.

Por último, el incremento de personas e instituciones colaboradoras en la organización (secretaría y contenido) ha favorecido la dinámica general de las Jornadas. A todos ellos queremos mostrar nuestro agradecimiento al hacer posible con su trabajo la celebración y ei resultado de las mismas.

$\mathbf{M}^{\mathrm{a}}$ Carmen Gómez Pascual Secretaria de organización 